Kiyoshi Ichihara*, Ferruccio Ceriotti, Tran Huu Tam, Shigeo Sueyoshi, Priscilla M.K. Poon, Mee Ling Thong, Yasushi Higashiuesato, Xuejing Wang, Hiromi Kataoka, Akemi Matsubara, Shu-Chu Shiesh, Dewi Muliaty, Jeong-Ho Kim, Masakazu Watanabe, Christopher W.K. Lam, Lothar Siekmann, Joseph B. Lopez, Mauro Panteghini and on behalf of the Committee on Reference Intervals and Decision Limits, International Federation for Clinical Chemistry and Laboratory Medicine, and the Science Committee for the Asia-Pacific Federation of Clinical Biochemistry

\title{
The Asian project for collaborative derivation of reference intervals: (1) strategy and major results of standardized analytes
}

\begin{abstract}
Background: A multicenter study conducted in Southeast Asia to derive reference intervals (RIs) for 72 commonly measured analytes (general chemistry, inflammatory markers, hormones, etc.) featured centralized measurement to clearly detect regionality in test results. The results of 31 standardized analytes are reported, with the remaining analytes presented in the next report.

Method: The study included 63 clinical laboratories from South Korea, China, Vietnam, Malaysia, Indonesia, and seven areas in Japan. A total of 3541 healthy individuals aged 20-65 years (Japan 2082, others 1459) were recruited mostly from hospital workers using a well-defined common protocol. All serum specimens were transported to Tokyo at $-80^{\circ} \mathrm{C}$ and collectively measured using reagents from four manufacturers. Three-level nested ANOVA was used to quantitate variation (SD) of test results due to region, sex, and age. A ratio of SD for a given factor over residual SD (representing net between-individual variations) (SDR) exceeding 0.3 was considered significant. Traceability of RIs was ensured by recalibration using value-assigned reference materials. RIs were derived parametrically.
\end{abstract}

Results: SDRs for sex and age were significant for 19 and 16 analytes, respectively. Regional difference was significant for 11 analytes, including high density lipoprotein (HDL)-cholesterol and inflammatory markers. However, when the data were limited to those from Japan, regionality was not observed in any of the analytes. Accordingly, RIs were derived with or without partition by sex and region.

Conclusions: RIs applicable to a wide area in Asia were established for the majority of analytes with traceability to reference measuring systems, whereas regional partitioning was required for RIs of the other analytes.
Keywords: common reference interval; multicenter study; nested ANOVA; regionality; standardization.

\section{Non-standard abbreviations}

Alb, albumin; ALP, alkaline phosphatase; ALT, alanine aminotransferase; AMY, amylase; AST, aspartate aminotransferase; BC, Beckman Coulter; BMI, body mass index; C3, complement component 3; C4, complement component 4; Ca, total calcium; CK, creatine kinase; $\mathrm{Cl}$, chloride; CRE, creatinine; CRM470, certified reference material 470; CRP, C-reactive protein; E2, estradiol; GGT, $\gamma$-glutamyltransferase; Glu, glucose; Hb, hemoglobin; HDL-C, high density lipoprotein-cholesterol; IgA, immunoglobulin A; IgG, immunoglobulin G; IgM, immunoglobulin M; JCTLM, Joint Committee on Traceability of Laboratory Medicine; JSCC, Japan Society of Clinical Chemistry; K, potassium; LD, lactate dehydrogenase; LDL-C, low density lipoprotein-cholesterol; MCV, mean corpuscular volume; Na, sodium; RI, reference interval; TCho, total cholesterol; Tf, transferrin; TG, triglycerides; TTR, transthyretin (prealbumin); UA, uric acid.

*Corresponding author: Kiyoshi Ichihara, MD, PhD, Department of Clinical Laboratory Sciences, Faculty of Health Sciences, Yamaguchi University Graduate School of Medicine, Minami-Kogushi 1-1-1, Ube, 755-8505 Japan, Phone: +81 836 222884, Fax: +81 836355213 , E-mail: ichihara@yamaguchi-u.ac.jp

Ferruccio Ceriotti: Diagnostica e Ricerca San Raffaele, San Raffaele Scientific Institute, Milan, Italy

Tran Huu Tam: Center for Standardization and QC in Medical Laboratories, Ho Chi Minh City, Vietnam 
Shigeo Sueyoshi: Clinical Laboratory, Chiba Cardiovascular Center, Ichihara, Japan

Priscilla M.K. Poon: Department of Chemical Pathology, Chinese University of Hong Kong, Hong Kong

Mee Ling Thong: Department of Laboratory and Blood Services, National Heart Institute, Kuala Lumpur, Malaysia

Yasushi Higashiuesato: Clinical Laboratory, University of the Ryukyus, Graduate School of Medicine, Okinawa, Japan

Xuejing Wang: Department of Clinical Laboratory Medicine, Peking University First Hospital, Beijing, China

Hiromi Kataoka: Center of Medical Information Science, Kochi Medical School, Kochi, Japan

Akemi Matsubara: Clinical Laboratory, Hiroshima University Hospital, Hiroshima, Japan

Shu-Chu Shiesh: Department of Medical Laboratory Science and Biotechnology, National Cheng Kung University, Tainan, Taiwan Dewi Muliaty: Prodia Clinical Laboratory, Jakarta, Indonesia Jeong-Ho Kim: Department of Laboratory Medicine, Yonsei University College of Medicine, Seoul, Korea

Masakazu Watanabe: Beckman Coulter Japan Co., Tokyo, Japan Christopher W. K. Lam: Macau Institute for Applied Research in Medicine and Health, Macau University of Science and Technology, Macau

Lothar Siekmann: Institute of Clinical Chemistry and Pharmacology, Universitätsklinikum, Bonn, Germany

Joseph B. Lopez: Department of Biomedical Sciences, MAHSA University College, Kuala Lumpur, Malaysia

Mauro Panteghini: Centre for Metrological Traceability in Laboratory Medicine (CIRME), University of Milano, Milan, Italy

\section{Introduction}

The global standardization of laboratory tests for major analytes has been achieved by the efforts of the International Federation of Clinical Chemistry and Laboratory Medicine (IFCC) and its national member organizations [1]. However, reference intervals (RIs) still frequently differ from one laboratory to another. According to the directive on in vitro diagnostic medical devices of the European Union [2], diagnostic manufacturers are now requested to supply their clients with appropriate RIs attached to their reagents, and the ISO 15189 standard for clinical laboratory accreditation claims that the laboratory should periodically re-evaluate its RIs [3]. However, despite these requirements, there have been only a limited number of attempts to conduct appropriate multicenter studies to achieve this goal [4-6]. Most studies to date were conducted by gathering test results of healthy donors' specimens measured at each participating laboratory. This relies on two main assumptions: comparability of assay results across different laboratories and lack of regionality in test results. Validation of these assumptions, however, is not easy and thus, the interpretation of RIs is not straightforward.

One multicenter study was conducted in six Asian cites by the Committee on Plasma Proteins (C-PP) of the IFCC Scientific Division (IFCC-SD) in 2000 to derive common reference intervals for 14 major serum proteins [7] whose standardization had almost been achieved by the availability of certified reference material 470 (CRM470) produced under the direction of the C-PP [8]. This study adopted a different approach, that of centralized measurement, by sending all of the specimens to one center in Japan and measuring them collectively in a single laboratory. Unexpectedly, it revealed large interregion variation for many analytes belonging to the class of inflammatory markers, such as IgG, C3, and CRP [7]. To confirm these findings, another study was conducted in 2006 with the participation of six other centers belonging to the Asia Pacific Federation of Clinical Biochemistry (APFCB) [9]. A total of 560 healthy individuals, mainly those working in clinical laboratories, donated blood to allow measurement of 32 common analytes ( 22 biochemical analytes and 10 proteins). This second survey confirmed significant regionality in about one third of the analytes examined.

Prompted by these observations, this present third study was planned jointly by the C-PP, the Committee on Reference Intervals and Decision Limits (C-RIDL) of the IFCC-SD, and by the Scientific Committee of the APFCB. The primary goal was to verify the possibility of establishing common RIs (i.e., RIs that can be applied to the entire population, independently from the analytical method in use [10]) for the standardized analytes by ensuring traceability of test results. To do this, we collected samples from several relatively homogenous groups of individuals in Japan and in Southeast Asia together with a considerable amount of information allowing us to put in evidence regarding possible effects due to environment and/or lifestyle. Thus, we were able to investigate biological sources of variations in each analyte, including the presence of differences in RIs related to geographical areas (regionality). Our work was not limited to well-standardized analytes but also included non-standardized analytes mostly measured by immunoassays, such as tumor markers, and hormones. To achieve these goals, we again adopted the centralized measurement approach to eliminate assay-related variations. For clarity of presentation, we report herein the results limited to 31 standardized analytes and leave results of other analytes to the next report. 


\section{Materials and methods}

\section{Participating centers}

Invitations to the study together with its protocol were sent to clinical laboratories of major hospitals and commercial laboratories in countries belonging to the APFCB. Fifteen laboratories located in Seoul, Beijing, Taipei, Tainan, Ho Chi Minh City, Kuala Lumpur, Hong Kong, Macau, and Jakarta accepted the invitation. In Japan, collaboration was obtained nationwide from 47 laboratories. All laboratories are listed in the Acknowledgments.

\section{Target population and sample size}

We limited the population mainly to those working in clinical laboratories or hospitals to eliminate the potential influence of differences in job profiles on the test results. A minimum sample size from each local area was set at 120 samples to gain adequate statistical power to detect differences among areas. Usually two or more laboratories from each city collaborated to achieve the target number. The age range of donors was set at 20-65 years. The utmost effort was made to ensure an even distribution of age and an equal male to female ratio.

The following exclusion criteria were applied at the time of recruitment: 1) body mass index (BMI) $\geq 28 \mathrm{~kg} / \mathrm{m}^{2} ; 2$ ) average daily consumption of ethanol $\geq 75 \mathrm{~g}$ [this amount of alcohol intake is rather high, but it was decided to leave it as a general criterion to evaluate the effect of alcohol assumption and to adopt specific exclusion criteria for analytes known to be influenced by alcohol intake (e.g., ALT, GGT, etc.)], 3) cigarette smoking >20/day, 4) regular medication for chronic disease (diabetes mellitus, hypertension, hyperlipidemia, allergic disorders, etc.), 5) recent ( $\leq 2$ weeks) recovery from acute illness or surgery requiring hospitalization, 6) known hepatitis B or C virus carrier, and 7) pregnancy or within 1 year after childbirth.

For analysis of sources of variation for each analyte, a healthstatus survey was conducted via questionnaire to obtain information on BMI, ABO blood type, eating patterns, recent episodes of infection or allergy, menstrual status, and other factors. Written informed consent was obtained from each volunteer. The specimens, the test results, and questionnaires were processed anonymously by assigning sequential numbers on arrival to each individual. After completion of measurement for major analytes, the test results together with newly derived sex-specific RIs were reported confidentially.

The study was approved by the Ethical Committee of Yamaguchi University Graduate School of Medical Sciences in December, 2008. The other collaborative laboratories followed their own rules in taking part in the study.

\section{Collection of blood samples}

Prior to blood sampling, each volunteer was requested to avoid strenuous physical exertion/exercise during the preceding 4 days, excessive eating or drinking the night before, and to fast overnight at least for $10 \mathrm{~h}$.

Venipuncture was done between $7 \mathrm{am}$ and $10 \mathrm{am}$ after the donor sat quietly for at least $15 \mathrm{~min}$ to avoid variations of test results due to physical activity and postural influence. Blood in the amount of $17 \mathrm{~mL}$ was drawn from each donor. All participating laboratories received the same blood drawing set containing the vacuum sampling system, provided by Becton-Dickinson Corp. (BD), (Franklin Lakes, NJ, USA), including BD Vacutainer SST tubes (capacity: $8.5 \mathrm{~mL}$ ) containing a clot-activator and CryoTube cryovials (capacity: $4.8 \mathrm{~mL}$ ) (Nunc, Roskilde, Denmark). After blood collection, the tubes were rocked $180^{\circ}$ at least five times, and centrifugation for serum separation was done within $2 \mathrm{~h}$ of collection. The separated serum was transferred and aliquoted immediately to the freezing vials for storage at $-80^{\circ} \mathrm{C}$. As an adjunct test, a peripheral blood count (CBC) in EDTA-2K blood was performed locally. The results were used as criteria to exclude those who were anemic. The CBC test results were obtained from 1978 individuals in laboratories that used the BeckmanCoulter hematology analyzer [Beckman Coulter Inc. (BC), Brea, CA, USA] for their routine assays.

\section{Target analytes and measurements}

Seventy-two analytes (lipids, electrolytes, enzymes, tumor markers, hormones, vitamins, etc.) were targeted for collective measurements. Assay methods, reference materials, and their precisions for the 31 standardized analytes included in this report are listed in Table 1. The auto-analyzer used in the centralized assays was the UniCel DxC (BC). Enzyme activities except for amylase (AMY) were measured in duplicate, one by the IFCC- and the other by JSCC (Japan Society for Clinical Chemistry)-recommended method. The assays were done at BC Japan's Central Laboratory located in Ariake, Tokyo, using the manufacturer's reagents, calibrators, and controls, unless otherwise indicated.

For quality control monitoring, a set of four fresh-frozen pooled sera from healthy individuals, stored aliquoted at $-80^{\circ} \mathrm{C}$, were used to evaluate imprecision of assays. The average results of imprecision using these samples are listed in Table 1.

\section{Specimen transportation}

Serum specimens were packed in a styrene foam box container filled with dry ice and transported to the central laboratory by a domestic express courier service within Japan. Outside Japan, the sample containers were picked up from each laboratory by the coordinator of this study and transported to Japan by airplane, carried as check-in baggage.

\section{Verification of traceability}

Reference methods and/or materials for the 31 analytes are available in the database of the Joint Committee on Traceability of Laboratory Medicine (JCTLM). We checked traceability to the corresponding reference system using the materials or methods listed in Table 1. When reference laboratory services were involved (enzymes and hormones), a set of three fresh-frozen $\left(-80^{\circ} \mathrm{C}\right)$ pooled sera for each enzyme (AST, ALT, LD, GGT, and CK), or five to nine levels of lyophilized specimens (for the four steroid hormones), were first value-assigned by the reference laboratories (see Acknowledgments) and then used to check alignment of the analytical systems used for obtaining reference 
Table 1 List of assay methods and assay imprecision near the mid-normal ranges.

\begin{tabular}{|c|c|c|c|c|c|c|}
\hline Abbr & Analytes & Method & Reagent & Traceability & $\begin{array}{l}\text { Within- } \\
\text { day CV }\end{array}$ & $\begin{array}{r}\text { Between- } \\
\text { day CV }\end{array}$ \\
\hline Alb & Albumin & $\begin{array}{l}\text { Timed endpoint bromcresol purple } \\
\text { method }\end{array}$ & Beckman Coulter & CRM470 & 1.53 & 1.37 \\
\hline Urea & Urea & Enzymatic rate method & Beckman Coulter & JCCRM521 & 1.54 & 2.46 \\
\hline UA & Uric acid & Timed endpoint uricase POD method & Beckman Coulter & JCCRM521, 21 & 0.68 & 1.11 \\
\hline CRE & Creatinine & Enzymatic method & Beckman Coulter & JCCRM521 & 2.41 & 2.97 \\
\hline $\mathrm{Na}$ & Sodium & Indirect potentiometry & Beckman Coulter & JCCRM111, 321 & 0.22 & 0.91 \\
\hline K & Potassium & Indirect potentiometry & Beckman Coulter & JCCRM111, 321 & 0.46 & 0.89 \\
\hline $\mathrm{Cl}$ & Chloride & Indirect potentiometry & Beckman Coulter & JCCRM111, 321 & 0.25 & 0.72 \\
\hline $\mathrm{Ca}$ & Total serum calcium & Indirect potentiometry & Beckman Coulter & JCCRM321 & 0.57 & 1.26 \\
\hline Glu $^{d}$ & Glucose & Timed endpoint hexokinase method & Beckman Coulter & JCCRM521 & 0.77 & 1.26 \\
\hline TCho & Total cholesterol & Timed endpoint enzyme colorimetry & Beckman Coulter & JCCRM223 & 1.20 & 0.74 \\
\hline TG & Triglyceride & Timed endpoint enzyme colorimetry & Beckman Coulter & JCCRM223, 224 & 1.21 & 0.80 \\
\hline HDL-C & HDL-cholesterol & Timed endpoint direct method & Beckman Coulter & JCCRM223, 224 & 0.99 & 1.61 \\
\hline LDL-C & LDL-cholesterol & Timed endpoint direct method & Beckman Coulter & JCCRM224 & 1.17 & 1.31 \\
\hline AST & $\begin{array}{l}\text { Aspartate } \\
\text { aminotransferase }\end{array}$ & IFCC recommended method & Beckman Coulter & JCTLM ref lab ${ }^{b}$ & 2.22 & 0.73 \\
\hline AST-JSCC & & JSCC recommended method & Beckman Coulter & JCCLS CRM-001b & 3.40 & 0.37 \\
\hline ALT & Alanine aminotransferase & IFCC recommended method & Beckman Coulter & JCTLM ref lab ${ }^{b}$ & 2.88 & 1.27 \\
\hline ALT-JSCC & & JSCC recommended method & Beckman Coulter & JCCLS CRM-001b & 3.44 & 0.44 \\
\hline LD & Lactate dehydrogenase & IFCC recommended method & Beckman Coulter & JCTLM ref lab ${ }^{b}$ & 1.42 & 0.29 \\
\hline LD-JSCC & & JSCC recommended method & Beckman Coulter & JCCLS CRM-001b & 1.93 & 0.80 \\
\hline ALP & Alkaline phosphatase & IFCC reccommended method & Beckman Coulter & - & 1.91 & 2.60 \\
\hline ALP-JSCC & & JSCC recommended method ${ }^{a}$ & Beckman Coulter & JCCLS CRM-001b & 1.45 & 0.83 \\
\hline GGT & $\gamma$-glutamyltransferase & IFCC recommended method & Beckman Coulter & JCTLM ref lab ${ }^{b}$ & 2.14 & 4.63 \\
\hline GGT-JSCC & & JSCC recommended method & Beckman Coulter & JCCLS CRM-001b & 1.60 & 1.86 \\
\hline CK & Creatine kinase & IFCC recommended method & Beckman Coulter & JCTLM ref lab ${ }^{b}$ & 0.86 & 0.88 \\
\hline CK-JSCC & & JSCC recommended method & Beckman Coulter & JCCLS CRM-001b & 1.22 & 0.91 \\
\hline AMY & Amylase & JSCC recommended method & Beckman Coulter & JCCLS CRM-001b & 0.89 & 0.61 \\
\hline $\lg G$ & Immunoglobulin G & Turbidimetric method & Nittobo Medical & CRM470 & 0.63 & 1.76 \\
\hline $\lg A$ & Immunoglobulin A & Turbidimetric method & Nittobo Medical & CRM470 & 1.76 & 3.40 \\
\hline $\lg M$ & Immunoglobulin M & Turbidimetric method & Nittobo Medical & CRM470 & 1.09 & 1.46 \\
\hline $\mathrm{C} 3$ & Complement component 3 & Turbidimetric method & Beckman Coulter & CRM470 & 0.81 & 1.77 \\
\hline $\mathrm{C} 4$ & Complement component 4 & Turbidimetric method & Beckman Coulter & CRM470 & 2.04 & 2.82 \\
\hline CRP & C-reactive protein & Latex immunoturbidimetric method & Nittobo Medical & CRM470 & 0.68 & 2.29 \\
\hline TTR & Transthyretin(prealbumin) & Turbidimetric method & Beckman Coulter & CRM470 & 1.72 & 2.98 \\
\hline Tf & Transferrin & Turbidimetric method & Beckman Coulter & CRM470 & 2.65 & 3.61 \\
\hline Testo & Testosterone & Chemiluminescent enzyme immunoassay & Beckman Coulter & JCTLM ref labc & 2.70 & 2.38 \\
\hline E2 & Estradiol & Chemiluminescent enzyme immunoassay & Beckman Coulter & JCTLM ref labc & 7.43 & 6.40 \\
\hline Prog & Progesterone & Chemiluminescent enzyme immunoassay & Beckman Coulter & JCTLM ref lab ${ }^{c}$ & 5.98 & 6.35 \\
\hline Cortisol & Cortisol & Chemiluminescent enzyme immunoassay & Beckman Coulter & JCTLM ref labc & 2.17 & 1.94 \\
\hline
\end{tabular}

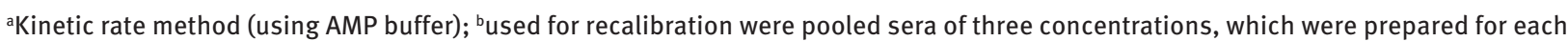
enzyme, and values were assigned by the reference measurement system (RMS); ' $u$ used for recalibration were lyophilized specimens of five to eight concentrations that were value-assigned by RMS; ${ }^{\mathrm{D}}$ The analyte was measured in specimens collected without addition of sodium fluoride. The result was used as one of the exclusion criteria.

values. Particularly, recalibration of test results was made based on the experimental relation between the assigned values and the test results (measured on three separate days, each in triplicate) obtained at BC's laboratory in Tokyo. When there were more than two levels of reference materials, the regression line to be used for recalibration was computed by use of the reduced major-axis regression method $[11,12]$. For reference materials that have just a single level, the ratio of the assigned value to average measured values was used for recalibration.

\section{Statistical analyses}

\section{Analyses for sources of variations and partitioning criterion}

The test results were evaluated by use of the same statistical procedures employed in the previous study [9]. In brief, sources (factors) of variations were analyzed by three-level nested ANOVA (3N-ANOVA). 
The factors considered were sex, region, age, and BMI. Primarily, the first three factors were analyzed; then, after separating data by sex, the analyses using the last three factors were performed. The magnitude of variation due to each factor is expressed as a standard deviation $(\mathrm{SD})$ : i.e., between-region $\mathrm{SD}\left(\mathrm{SD}_{\text {reg }}\right)$, between-sex $\mathrm{SD}\left(\mathrm{SD}_{\mathrm{sex}}\right)$, and between-age $\mathrm{SD}\left(\mathrm{SD}_{\text {age }}\right)$. In the analysis, regions were categorized into 14 areas ( 7 within Japan, 7 outside Japan), age was stratified into four groups (20-29, 30-39, 40-49, and 50-65 years), and BMI was separated into six categories by setting the following boundary values: 18 , $20,22,24$, and $26 \mathrm{~kg} / \mathrm{m}^{2}$.

The relative magnitude of each variable to that of the residual $\mathrm{SD}$ representing a net between-individual $\mathrm{SD}\left(\mathrm{SD}_{\text {net-btw-indiv }}\right)$, which roughly corresponds to one quarter of the width of the RI obtained after adjusting for the influence of age and sex, was computed as the SD ratio (SDR) by the following formula:

$$
\mathrm{SDR}_{\text {factor }}=\mathrm{SD}_{\text {factor }} / \mathrm{SD}_{\text {net-btw-indiv }}
$$

An SDR of $\geq 0.3$ was regarded as high, requiring partition of reference values by the factor [13]. Meanwhile, an SDR between 0.25 and 0.29 was considered moderate in degree. In the above computation, when the distribution of the test results was skewed and had a long upper tail, the values were logarithmically transformed before 3N-ANOVA was applied (as indicated in Table 2). In these cases, the back-transformed SD described elsewhere [9] was used in the above equation. In the analysis of regionality, when computing SDR for region $\left(\mathrm{SDR}_{\mathrm{reg}}\right)$, test results obtained within Japan were all combined, as were those from the two cities in Taiwan, and those from Hong Kong and Macau, respectively, as single regions. Meanwhile, test results from Seoul, Beijing, Ho Chi Minh City, Kuala Lumpur, and Jakarta were treated as separate regions; thus, the number of regions served for the analysis of $\mathrm{SDR}_{\mathrm{reg}}$ was eight in all.

\section{Derivation of reference intervals}

As a preliminary step to select appropriate reference individuals, those who had extreme values in analytes related to obesity, anemia, inflammation, and thyroid dysfunction as follows were excluded before the derivation of RIs: $\mathrm{Hb} \leq 96$ or $\geq 180 \mathrm{~g} / \mathrm{L}, \mathrm{MCV} \leq 70 \mathrm{fL}$, UA $\leq 95$ or $\geq 536 \mu \mathrm{mol} / \mathrm{L}$ ( $\leq 1.6$ or $\geq 9.0 \mathrm{mg} / \mathrm{dL}$ ), $\mathrm{TG}>3.96 \mathrm{mmol} / \mathrm{L}$ ( $350 \mathrm{mg} / \mathrm{dL}$ ), Glu $>6.95 \mathrm{mmol} / \mathrm{L}(125 \mathrm{mg} / \mathrm{dL})$, TCho $>8.04 \mathrm{mmol} / \mathrm{L}$ (311 mg/dL), ALT $>101 \mathrm{U} / \mathrm{L}$, GGT $>140 \mathrm{U} / \mathrm{L}$, AST $>80 \mathrm{U} / \mathrm{L}, \mathrm{CK}>800 \mathrm{U} / \mathrm{L}$, CRP $>20 \mathrm{mg} / \mathrm{L}$, FT4 >23.17 pmol/L (1.8 ng/dL), and TSH >14 mU/L. As for BMI, 65 (1.8\%) individuals exceeded the upper limit of $28 \mathrm{~kg} / \mathrm{m}^{2}$ set at the time of recruitment. In consideration of the imprecision of BMI and the availability of related information indicating obesity, the final exclusion criterion was set as a BMI of $\leq 14$ or $\geq 30 \mathrm{~kg} / \mathrm{m}^{2}$. Thus, 21 individuals were excluded by these BMI extremes.

The RIs were derived parametrically by use of modified Box-Cox transformation [14], which invariably succeeds in transforming reference values into those of Gaussian distribution as long as there are not many results that are below the detectable limits [13]. As a tertiary exclusion step to further refine the reference values, an iterative approach called the 'latent abnormal values exclusion' (LAVE) method was applied for the actual derivation of the RIs [7, 13, 14]. As exclusion criteria, the RIs of the following 13 analytes were used: Alb, Glb, UA, Glu, AST, ALT, LD, GGT, CK, TG, HDL-C, LDL-C, and CRP. In short, the initial RIs were derived analyte by analyte independently of the results of the others. From the second iteration, the RIs for the above exclusion criteria analytes obtained at the previous cycle of iteration were used to exclude individuals who had abnormal results in analytes other than the one being evaluated. In applying the exclusion criteria, the RIs were extended on both ends by $5 \%$ of the interval, or (upper limit-lower limit) $\times 0.05$. Therefore, when the test results are normally distributed, results outside of the mean $\pm 2.16 \mathrm{SD}$ (total of $3 \%$ on two tails) are regarded as abnormal. This adjustment was made to avoid excluding too many individuals unnecessarily. The computation was continued until the RIs of all the analytes became stable.

Statistical analyses and 3N-ANOVA was performed with general purpose statistical software StatFlex for Windows Ver. 6.0 (Artech, Osaka, Japan). Original software named 'Reference Master' developed by the first author was employed to derive RIs based on the LAVE principle and parametric methods.

\section{Results}

\section{Profile of the subjects}

The demographic profile of the participants from each city is summarized in Table 3. The tabulation was made after deleting those with extreme values based on the criteria described in the Methods. As a whole, there were more females than males (1959 vs. 1582). The information on ethnicity was not obtained in Japan, Korea, and China with known homogeneity of the target population in the medical facilities involved. The ethnic composition in other countries was as follows: in Kuala Lumpur: Chinese 36\%, Malay 45\%, Indian 13\%; in Ho Chi Minh City: Vietnamese (Kinh) 98\%; in Jakarta: Malay 86\%, Chinese 13\%.

The between-region differences in age, BMI, and levels of smoking and alcohol use were analyzed by one-way ANOVA separately for each sex. The ratios of pure component of between-region SD divided by residual SD representing between-individual SD in males (females) were $0.10(0.10)$ for age, $0.21(0.22)$ for BMI, 0.18 (0.17) for smoking, and $0.63(0.55)$ for alcohol, respectively. The ratio has the same implication as the SDR described above as a means of computing the magnitude of a given variation source. If we set 0.30 as the practically significant effect size of each factor, only the level of alcohol use was apparently different across the Asian cities, reflecting differences in religion and culture.

The job profile of the reference individuals $(n=3314)$ after the selection process is shown in Supplementary Table 1 which accompanies the article at http://www.degruyter. com/view/j/cclm.2013.51.issue-7/issue-files/cclm.2013.51. issue-7.xml. Those individuals who belonged to the medical institutions and those who did not accounted for 83.4\% (62.9\%-91.5\%) and 9.9\% (2.7\%-27.3\%), respectively, 


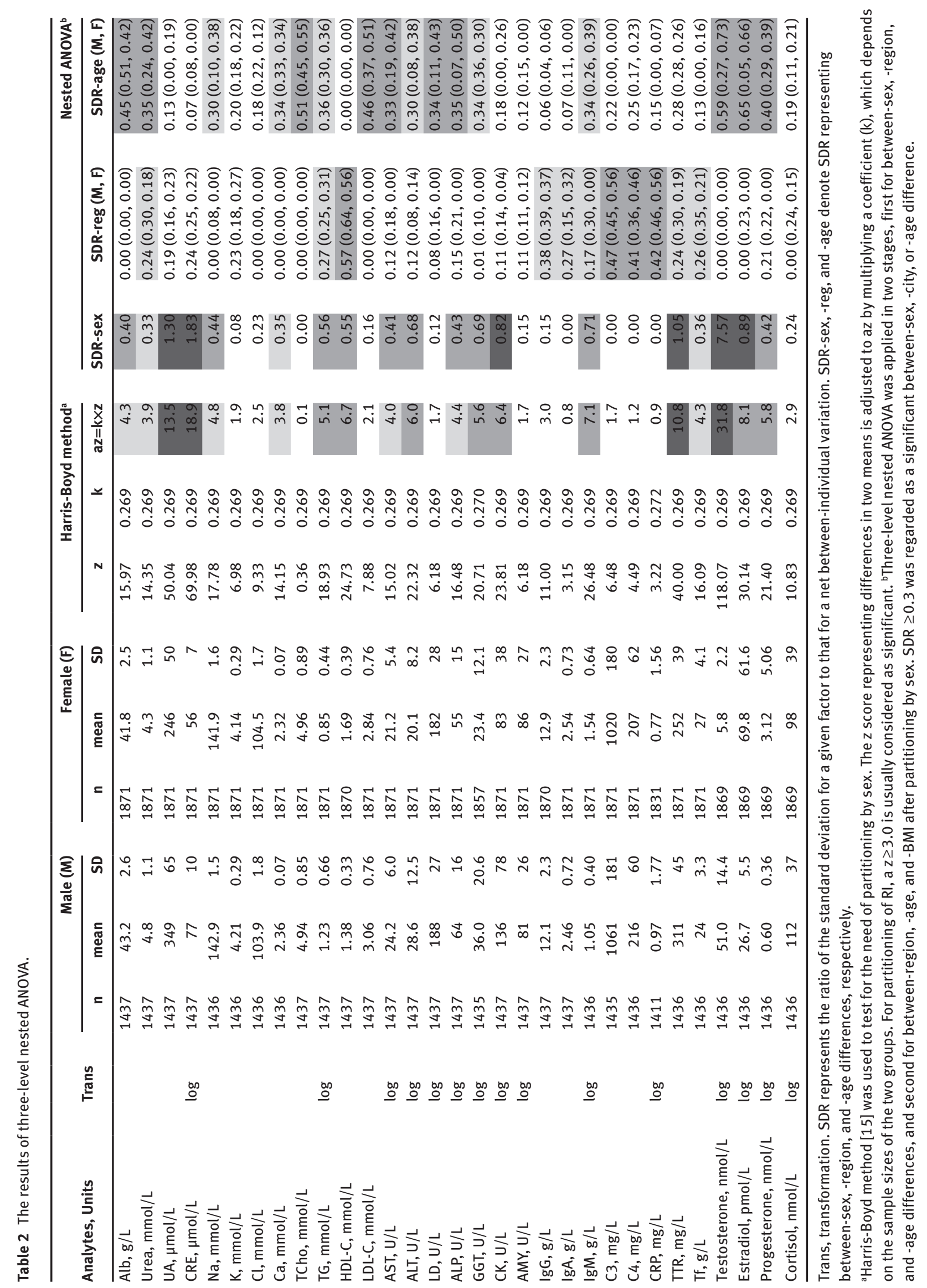


Table 3 Demography of the healthy individuals recruited.

\begin{tabular}{|c|c|c|c|c|c|c|c|c|c|c|}
\hline \multirow[t]{2}{*}{ Male } & \multirow[t]{2}{*}{$\mathbf{N}_{0}$} & \multirow[t]{2}{*}{$\mathbf{N}$} & \multicolumn{2}{|r|}{ Age } & \multicolumn{2}{|r|}{ BMI } & \multicolumn{2}{|c|}{ Tobacco } & \multicolumn{2}{|c|}{ Alcohol } \\
\hline & & & Me & $95 \% \mathrm{Cl}$ & $\mathrm{Me}$ & $95 \% \mathrm{Cl}$ & No & Yes & Rare & Yes \\
\hline \multirow[t]{2}{*}{ North Japan } & 157 & 146 & 38 & $23-60$ & 22.3 & $17.7-27.8$ & 97 & 49 & 51 & 95 \\
\hline & & & & & & & $(66 \%)$ & (34\%) & $(35 \%)$ & $(65 \%)$ \\
\hline \multirow[t]{2}{*}{ Tokyo } & 130 & 119 & 36 & $22-59$ & 22.4 & $17.3-27.5$ & 87 & 31 & 46 & 72 \\
\hline & & & & & & & (74\%) & $(26 \%)$ & (39\%) & (61\%) \\
\hline \multirow[t]{2}{*}{ Central Japan } & 127 & 109 & 41 & $23-58$ & 22.3 & $17.6-27.8$ & 81 & 28 & 45 & 64 \\
\hline & & & & & & & $(74 \%)$ & $(26 \%)$ & (41\%) & (59\%) \\
\hline \multirow[t]{2}{*}{ Osaka } & 116 & 110 & 40 & $21-61$ & 22.4 & $17.9-27.8$ & 85 & 25 & 33 & 77 \\
\hline & & & & & & & (77\%) & $(23 \%)$ & $(30 \%)$ & $(70 \%)$ \\
\hline \multirow[t]{2}{*}{ West Japan } & 185 & 176 & 39 & $23-60$ & 22.0 & $18.4-26.8$ & 129 & 46 & 59 & 116 \\
\hline & & & & & & & (74\%) & $(26 \%)$ & (34\%) & $(66 \%)$ \\
\hline \multirow[t]{2}{*}{ South Japan } & 136 & 127 & 36 & $20-60$ & 22.0 & $17.7-27.9$ & 76 & 47 & 48 & 76 \\
\hline & & & & & & & $(62 \%)$ & $(38 \%)$ & (39\%) & $(61 \%)$ \\
\hline \multirow[t]{2}{*}{ Okinawa } & 96 & 92 & 35 & $21-59$ & 22.8 & $17.9-27.7$ & 69 & 23 & 26 & 66 \\
\hline & & & & & & & $(75 \%)$ & $(25 \%)$ & $(28 \%)$ & $(72 \%)$ \\
\hline \multirow[t]{2}{*}{ Seoul } & 60 & 56 & 38 & $23-58$ & 22.8 & $19.2-27.5$ & 36 & 20 & 24 & 32 \\
\hline & & & & & & & $(64 \%)$ & $(36 \%)$ & (43\%) & $(57 \%)$ \\
\hline \multirow[t]{2}{*}{ Beijing } & 59 & 51 & 36 & $23-58$ & 24.4 & $17.3-28.2$ & 38 & 13 & 41 & 10 \\
\hline & & & & & & & $(75 \%)$ & $(25 \%)$ & $(80 \%)$ & $(20 \%)$ \\
\hline \multirow[t]{2}{*}{ Taiwan } & 145 & 137 & 36 & $22-60$ & 22.8 & $17.4-27.3$ & 118 & 19 & 121 & 16 \\
\hline & & & & & & & $(86 \%)$ & $(14 \%)$ & $(88 \%)$ & $(12 \%)$ \\
\hline \multirow[t]{2}{*}{ Hong Kong } & 118 & 105 & 41 & $23-59$ & 23.7 & $18.2-27.5$ & 90 & 15 & 90 & 15 \\
\hline & & & & & & & $(86 \%)$ & $(14 \%)$ & $(86 \%)$ & (14\%) \\
\hline \multirow[t]{2}{*}{$\mathrm{HCMC}$} & 131 & 107 & 33 & $22-58$ & 22.1 & $17.6-27.7$ & 79 & 28 & 70 & 36 \\
\hline & & & & & & & $(74 \%)$ & $(26 \%)$ & $(66 \%)$ & (34\%) \\
\hline \multirow[t]{2}{*}{ Kuala Lumpur } & 63 & 52 & 31 & $20-60$ & 23.2 & $17.8-28.4$ & 37 & 15 & 49 & 0 \\
\hline & & & & & & & (71\%) & $(29 \%)$ & $(100 \%)$ & $(0 \%)$ \\
\hline \multirow[t]{2}{*}{ Jakarta } & 59 & 51 & 39 & $24-63$ & 23.8 & $18.4-28.7$ & 37 & 14 & 51 & 0 \\
\hline & & & & & & & (73\%) & $(27 \%)$ & $(100 \%)$ & $(0 \%)$ \\
\hline \multirow[t]{2}{*}{ Sum } & 1582 & 1438 & 37 & $21-59$ & 22.6 & $17.7-27.8$ & 1059 & 373 & 754 & 675 \\
\hline & & & & & & & $(74 \%)$ & $(26 \%)$ & (53\%) & (47\%) \\
\hline
\end{tabular}

\begin{tabular}{|c|c|c|c|c|c|c|c|c|c|c|c|c|c|}
\hline \multirow[t]{2}{*}{ Female } & \multirow[t]{2}{*}{$\mathrm{N}_{0}$} & \multirow[t]{2}{*}{$\mathbf{N}$} & \multicolumn{2}{|r|}{ Age } & \multicolumn{2}{|r|}{ BMI } & \multicolumn{2}{|c|}{ Tobacco } & \multicolumn{2}{|c|}{ Alcohol } & \multicolumn{3}{|c|}{ Menopause } \\
\hline & & & $\mathrm{Me}$ & $95 \% \mathrm{Cl}$ & Me & $95 \% \mathrm{Cl}$ & No & Yes & Rare & Yes & No & Yes & \\
\hline \multirow[t]{2}{*}{ North Japan } & 186 & 184 & 37 & $22-59$ & 19.8 & $16.4-26.1$ & 160 & 23 & 107 & 77 & 144 & 37 & \\
\hline & & & & & & & $(87 \%)$ & $(13 \%)$ & (58\%) & $(42 \%)$ & $(80 \%)$ & $(20 \%)$ & \\
\hline \multirow[t]{2}{*}{ Tokyo } & 183 & 177 & 35 & $22-58$ & 20.9 & $17.4-28.1$ & 161 & 16 & 109 & 68 & 150 & 23 & \\
\hline & & & & & & & (91\%) & (9\%) & $(62 \%)$ & $(38 \%)$ & $(87 \%)$ & (13\%) & \\
\hline \multirow[t]{2}{*}{ Central Japan } & 188 & 186 & 36 & $22-57$ & 19.8 & $16.2-24.9$ & 176 & 9 & 116 & 69 & 150 & 29 & \\
\hline & & & & & & & (95\%) & $(5 \%)$ & $(63 \%)$ & $(37 \%)$ & $(84 \%)$ & $(16 \%)$ & \\
\hline \multirow[t]{2}{*}{ Osaka } & 136 & 133 & 38 & $21-60$ & 20.8 & $16.4-26.7$ & 119 & 5 & 84 & 42 & 100 & 24 & \\
\hline & & & & & & & (96\%) & $(4 \%)$ & $(67 \%)$ & $(33 \%)$ & $(81 \%)$ & (19\%) & \\
\hline \multirow[t]{2}{*}{ West Japan } & 232 & 224 & 37 & $23-60$ & 19.8 & $16.6-24.6$ & 217 & 7 & 144 & 79 & 172 & 45 & \\
\hline & & & & & & & (97\%) & (3\%) & $(65 \%)$ & $(35 \%)$ & (79\%) & $(21 \%)$ & \\
\hline \multirow[t]{2}{*}{ South Japan } & 113 & 107 & 37 & $20-58$ & 20.1 & $17-25.9$ & 98 & 9 & 78 & 29 & 89 & 16 & \\
\hline & & & & & & & (92\%) & (8\%) & (73\%) & $(27 \%)$ & $(85 \%)$ & (15\%) & \\
\hline \multirow[t]{2}{*}{ Okinawa } & 97 & 96 & 35 & $21-57$ & 20.7 & $17.6-26.6$ & 94 & 1 & 58 & 37 & 86 & 9 & \\
\hline & & & & & & & (99\%) & (1\%) & $(61 \%)$ & (39\%) & (91\%) & (9\%) & \\
\hline \multirow[t]{2}{*}{ Seoul } & 73 & 71 & 38 & $22-61$ & 20.9 & $17.7-27.6$ & 69 & 2 & 54 & 16 & 57 & 11 & \\
\hline & & & & & & & (97\%) & (3\%) & (77\%) & $(23 \%)$ & $(84 \%)$ & (16\%) & \\
\hline \multirow[t]{2}{*}{ Beijing } & 83 & 78 & 39 & $22-58$ & 22.0 & $17.6-26.9$ & 76 & 2 & 77 & 1 & 59 & 18 & 1 \\
\hline & & & & & & & (97\%) & (3\%) & (99\%) & (1\%) & (77\%) & $(23 \%)$ & \\
\hline \multirow[t]{2}{*}{ Taiwan } & 190 & 175 & 36 & $23-59$ & 20.7 & $16.8-27.3$ & 174 & 0 & 174 & 1 & 145 & 30 & 0 \\
\hline & & & & & & & (100\%) & $(0 \%)$ & (99\%) & (1\%) & (83\%) & (17\%) & \\
\hline
\end{tabular}


(Table 3 Continued)

\begin{tabular}{|c|c|c|c|c|c|c|c|c|c|c|c|c|c|}
\hline \multirow[t]{2}{*}{ Female } & \multirow[t]{2}{*}{$\mathrm{N}_{0}$} & \multirow[t]{2}{*}{$\mathbf{N}$} & \multicolumn{2}{|r|}{ Age } & \multicolumn{2}{|r|}{ BMI } & \multicolumn{2}{|c|}{ Tobacco } & \multicolumn{2}{|c|}{ Alcohol } & \multicolumn{3}{|c|}{ Menopause } \\
\hline & & & $\mathrm{Me}$ & $95 \% \mathrm{Cl}$ & $\mathrm{Me}$ & $95 \% \mathrm{Cl}$ & No & Yes & Rare & Yes & No & Yes & ? \\
\hline \multirow[t]{2}{*}{ Hong Kong } & 132 & 121 & 39 & $21-58$ & 21.1 & $17.5-28$ & 117 & 4 & 118 & 3 & 106 & 15 & 0 \\
\hline & & & & & & & (97\%) & (3\%) & (98\%) & $(2 \%)$ & (88\%) & (12\%) & \\
\hline \multirow[t]{2}{*}{ HCMC } & 170 & 166 & 33 & $22-53$ & 20.1 & $17-25.9$ & 165 & 1 & 162 & 3 & 152 & 13 & \\
\hline & & & & & & & (99\%) & (1\%) & (98\%) & $(2 \%)$ & (92\%) & (8\%) & \\
\hline \multirow[t]{2}{*}{ Kuala Lumpur } & 104 & 88 & 31 & $21-59$ & 21.1 & $16.8-27.7$ & 87 & 1 & 84 & 1 & 71 & 11 & \\
\hline & & & & & & & (99\%) & (1\%) & (99\%) & (1\%) & (87\%) & (13\%) & \\
\hline \multirow[t]{2}{*}{ Jakarta } & 72 & 70 & 39 & $21-60$ & 21.7 & $17.4-26.9$ & 69 & 1 & 70 & 0 & 55 & 15 & 0 \\
\hline & & & & & & & (99\%) & (1\%) & (100\%) & $(0 \%)$ & (79\%) & (21\%) & \\
\hline \multirow[t]{2}{*}{ Sum } & 1959 & 1876 & 36 & $21-59$ & 20.4 & $16.8-26.7$ & 1782 & 81 & 1435 & 426 & 1536 & 296 & 44 \\
\hline & & & & & & & (96\%) & $(4 \%)$ & (77\%) & $(23 \%)$ & (84\%) & (16\%) & \\
\hline
\end{tabular}

$\mathrm{Cl}$, confidence intervals; $\mathrm{Me}$, median; $\mathrm{N}_{0}$, the number of subjects before the secondary exclusion; $\mathrm{N}$, the number of subjects after the secondary exclusion.

of the reference individuals, whereas the remainder did not provide information on their job.

\section{Standardization of test results}

Excellent linearity between the test results and the values assigned to the reference materials with correlation coefficients very close to 1.0 were obtained for all the analytes that were measured in three or more levels (Supplementary Figure 1). The major axis regression was used to compute the regression line for recalibration. The results of reference materials measured at only a single level were used for recalibration by taking their ratio to the assigned values (Supplementary Table 2).

\section{Sources of variations and regionality of results}

The result of the 3N-ANOVA for all the analytes are summarized in Table 2 . The three factors analyzed were sex, region, and age. Those analytes indicated as 'log' in the 3rd column were transformed logarithmically before applying 3N-ANOVA. The level of SDR for sex $\left(\mathrm{SDR}_{\text {sex }}\right)$ was high $(\geq 0.3)$ for 19 analytes. In the table, the magnitude of between-sex difference quantified by 3N-ANOVA is compared with that by the Harris-Boyd method [12, 13]. The two approaches agreed quite well in almost all analytes. The SDRs for region $\left(\mathrm{SDR}_{\mathrm{reg}}\right)$ were high in five analytes: HDL-C, CRP, IgG, C3, and C4. SDRs for age $\left(\mathrm{SDR}_{\text {age }}\right)$ were high in 16 analytes.

Additionally, we noted unmatched regionality between the two sexes when we applied the 3N-ANOVA separately for males and females using the entire dataset, with inclusion of BMI as the third factor. The $\mathrm{SDR}_{\mathrm{reg}}$ computed for six analytes (urea, TG, IgA, IgM, Tf, and TTR) exceeded 0.3 only in either of the sexes (also shown in Supplementary Table 3A). As a whole, $\mathrm{SDR}_{\text {reg }}$ of 11 analytes exceeded 0.3 in either or both of the sexes. This finding implies the importance of separate analysis for both sexes to clearly identify regionality by use of 3N-ANOVA. When data was limited to those obtained from Japan (Supplementary Table 3B), there were no analytes that showed an SDR of $\geq 0$.3. Examples of regional differences for eight analytes with a high SDR reg score (urea, HDL-C, IgG, IgA, C3, C4, and CRP, and Tf) are illustrated in Figure 1. Similar figures for the entire group of results including the items from questionnaires are also available on-line as Supplementary Figure 2.

\section{Reference intervals}

RIs were derived from the reference individuals after the selection process in three ways: male plus female, male only, and female only, and by use of the modified Box-Cox formula. On the basis of the above results on regionality, when $\mathrm{SDR}_{\text {reg }}$ was $\geq 0.25$, RIs were computed in four ways: all Asians, Japanese, Chinese (Beijing+Taiwan+Hong Kong+Macau), and Southeast Asians (Ho Chi Minh City+Kuala Lumpur+Jakarta); otherwise, RIs were computed from the entire dataset (all Asians), as listed in Table 4.

In the derivation of RIs, we applied the tertiary exclusion procedure based on the LAVE method. Approximately $13 \%$ of the original data were excluded (3314 $\rightarrow$ roughly 2880 individuals) by the iterative optimization process. The effect of this tertiary exclusion was apparent only for analytes 

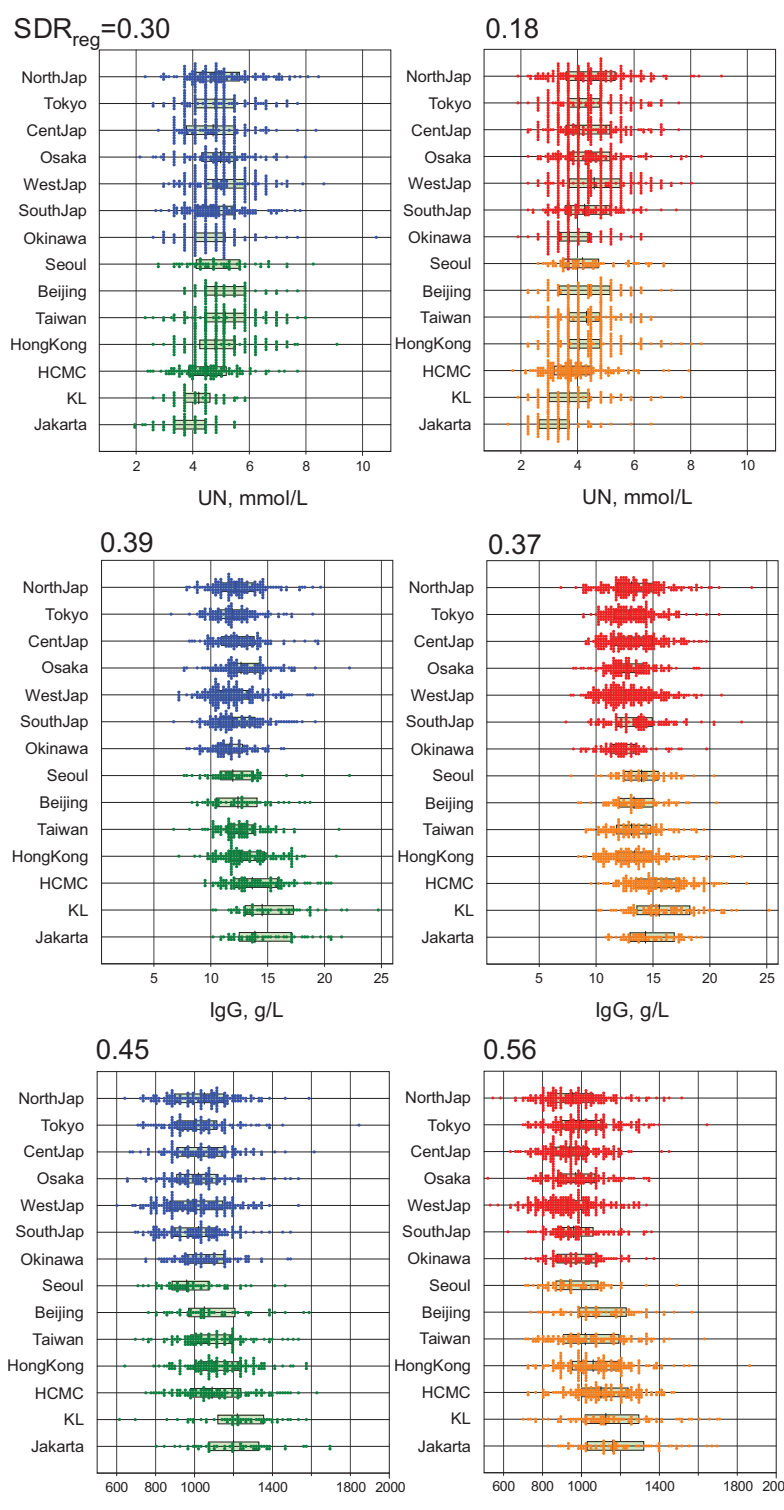

$\mathrm{C} 3, \mathrm{mg} / \mathrm{L}$

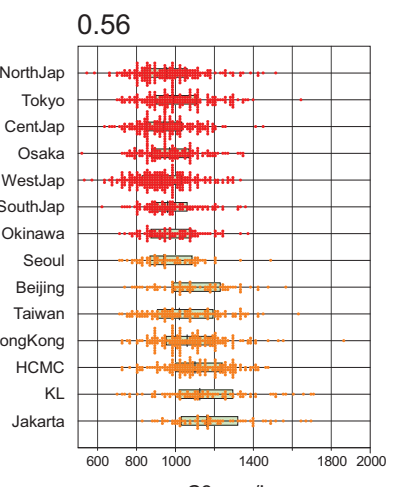

$\mathrm{C} 3, \mathrm{mg} / \mathrm{L}$

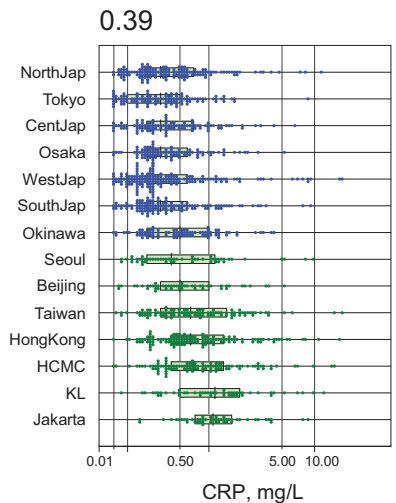

0.42

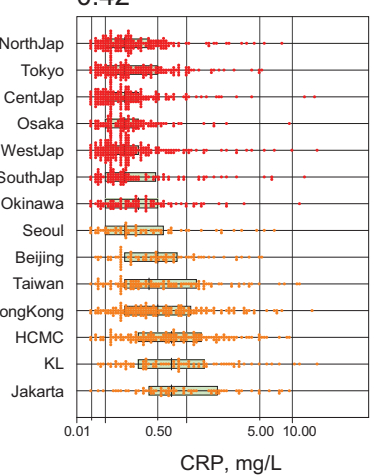

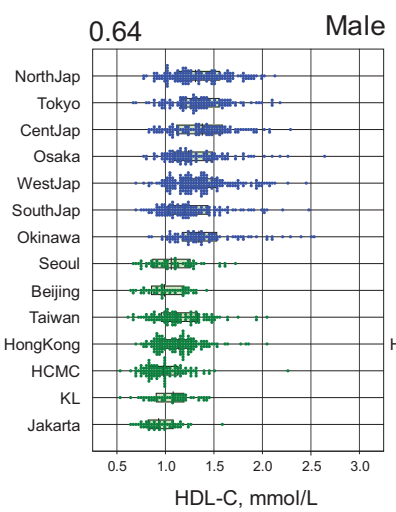
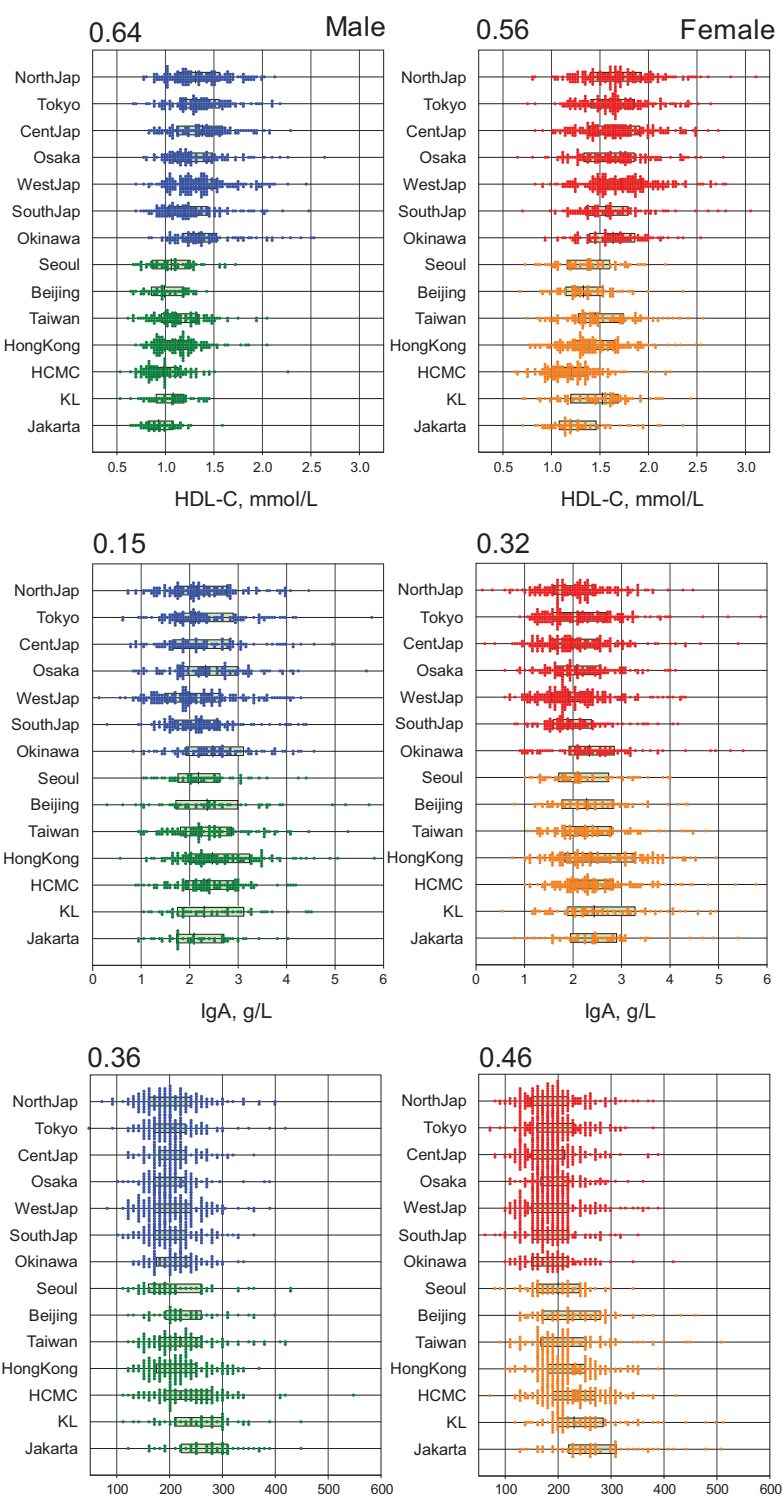

$\mathrm{C} 4, \mathrm{mg} / \mathrm{L}$

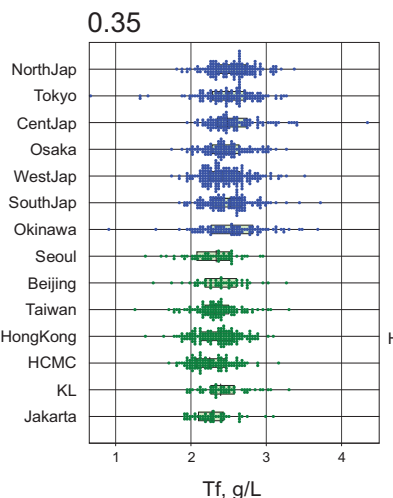

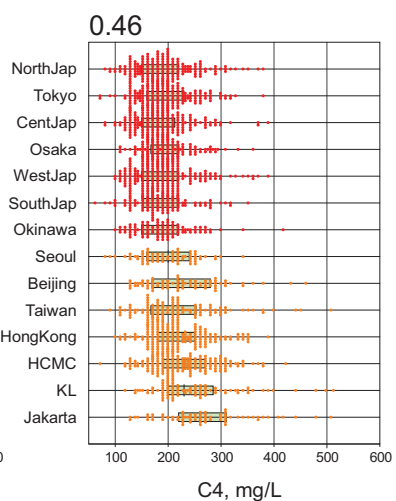

0.21

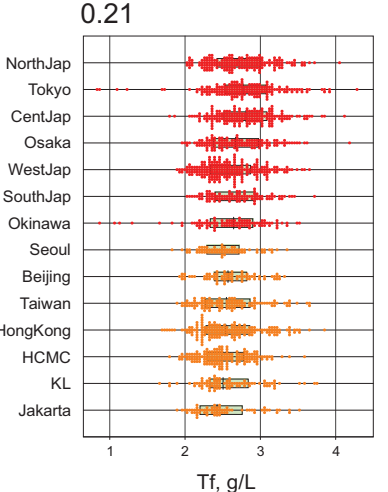

Figure 1 Examples of regional differences in test results observed in eight analytes.

Each panel consists of two figures, one for males (left) and one for females (right). Test results were sub-grouped into 14 areas: seven from Japan (blue, male; red, female) and another seven from East and Southeast Asia (green, male; orange, female). The regions were aligned from north to south. The box within each scattergram represents the central $50 \%$ range, and the vertical line in the middle of the box indicates the median. SDR reg displayed on top of each panel represents SD ratio of between-region variations to the pure between-individual variation. $S D R>0.3$ was considered as practically significant. 
Table 4 List of RIs derived.

\begin{tabular}{|c|c|c|c|c|c|c|c|c|c|c|c|c|c|c|}
\hline \multirow{2}{*}{$\frac{\text { International Unit }}{\text { Analytes, Units }}$} & \multirow[t]{2}{*}{ SDR-reg } & \multirow[t]{2}{*}{ Area } & \multirow[b]{2}{*}{$n$} & \multicolumn{3}{|c|}{ Male + Female } & \multirow[b]{2}{*}{$n$} & \multicolumn{3}{|r|}{ Male } & \multirow[b]{2}{*}{$\mathrm{n}$} & \multicolumn{3}{|c|}{ Female } \\
\hline & & & & LL & Me & UL & & LL & Me & UL & & LL & $\mathrm{Me}$ & UL \\
\hline Alb, g/L & 0.00 & Asia All & 2920 & 41 & 45 & 51 & 1298 & 41 & 46 & 51 & 1626 & 40 & 45 & 50 \\
\hline Urea, mmol/L & 0.24 & Asia All & 2888 & 2.7 & 4.4 & 7.1 & 1277 & 2.9 & 4.7 & 7.3 & 1608 & 2.6 & 4.1 & 6.8 \\
\hline $\mathrm{UA}, \mu \mathrm{mol} / \mathrm{L}$ & 0.19 & Asia All & & & & & 1293 & 223 & 347 & 471 & 1629 & 153 & 240 & 344 \\
\hline CRE, $\mu \mathrm{mol} / \mathrm{L}$ & 0.24 & Asia All & & & & & 1276 & 61.0 & 76.7 & 97.0 & 1611 & 41.9 & 55.8 & 70.6 \\
\hline $\mathrm{Na}, \mathrm{mmol} / \mathrm{L}$ & 0.00 & Asia All & 2886 & 139 & 142 & 146 & 1277 & 140 & 143 & 146 & 1606 & 139 & 142 & 145 \\
\hline $\mathrm{K}, \mathrm{mmol} / \mathrm{L}$ & 0.23 & Asia All & 2872 & 3.7 & 4.2 & 4.7 & 1270 & 3.7 & 4.2 & 4.7 & 1604 & 3.6 & 4.1 & 4.7 \\
\hline $\mathrm{Cl}, \mathrm{mmol} / \mathrm{L}$ & 0.00 & Asia All & 2889 & 101 & 104 & 108 & 1277 & 100 & 104 & 108 & 1611 & 101 & 104 & 108 \\
\hline $\mathrm{Ca}, \mathrm{mmol} / \mathrm{L}$ & 0.00 & Asia All & 2887 & 2.19 & 2.33 & 2.47 & 1276 & 2.21 & 2.35 & 2.49 & 1611 & 2.18 & 2.31 & 2.45 \\
\hline TCho, mmol/L & 0.00 & Asia All & 2889 & 3.47 & 4.85 & 6.73 & 1278 & 3.49 & 4.85 & 6.74 & 1611 & 3.48 & 4.83 & 6.80 \\
\hline \multirow[t]{4}{*}{$\mathrm{TG}, \mathrm{mmol} / \mathrm{L}$} & 0.27 & Asia All & 1551 & 0.41 & 0.81 & 2.20 & 588 & 0.49 & 1.00 & 2.77 & 961 & 0.39 & 0.73 & 1.67 \\
\hline & & Japanese & 1307 & 0.41 & 0.73 & 1.72 & 474 & 0.47 & 0.90 & 2.18 & 836 & 0.39 & 0.66 & 1.40 \\
\hline & & Chinese & 538 & 0.41 & 0.84 & 2.12 & 216 & 0.55 & 1.02 & 2.80 & 312 & 0.43 & 0.77 & 1.91 \\
\hline & & SE Asia & 422 & 0.47 & 0.99 & 3.05 & 159 & 0.53 & 1.24 & 3.38 & 252 & 0.50 & 0.87 & 2.50 \\
\hline \multirow[t]{4}{*}{$\mathrm{HDL}-\mathrm{C}, \mathrm{mmol} / \mathrm{L}$} & 0.57 & Asia All & 2915 & 0.94 & 1.52 & 2.43 & 1283 & 0.89 & 1.34 & 2.11 & 1628 & 1.07 & 1.67 & 2.55 \\
\hline & & Japanese & 1748 & 1.04 & 1.63 & 2.54 & 790 & 0.99 & 1.44 & 2.27 & 955 & 1.19 & 1.78 & 2.64 \\
\hline & & Chinese & 596 & 0.95 & 1.40 & 2.25 & 268 & 0.85 & 1.24 & 1.81 & 336 & 1.02 & 1.56 & 2.32 \\
\hline & & SE Asia & 483 & 0.85 & 1.28 & 2.10 & 197 & 0.81 & 1.11 & 1.61 & 285 & 0.98 & 1.43 & 2.24 \\
\hline LDL-C, $\mathrm{mmol} / \mathrm{L}$ & 0.00 & Asia All & 1561 & 1.65 & 2.83 & 4.55 & 594 & 1.83 & 2.94 & 4.85 & 969 & 1.62 & 2.75 & 4.46 \\
\hline AST, U/L & 0.12 & Asia All & 1872 & 14.4 & 21.0 & 32.3 & 803 & 15.8 & 22.8 & 34.8 & 1064 & 14.3 & 20.0 & 29.4 \\
\hline AST-JSCC, U/L & - & Japanese & 1715 & 15.2 & 21.3 & 30.8 & 769 & 16.1 & 22.9 & 32.9 & 953 & 14.7 & 20.3 & 28.0 \\
\hline ALT, U/L & 0.12 & Asia All & 1562 & 11.5 & 19.5 & 43.6 & 591 & 13.7 & 23.9 & 54.0 & 973 & 10.6 & 17.6 & 31.0 \\
\hline ALT-JSCC, U/L & - & Japanese & 1280 & 10.5 & 16.8 & 32.6 & 467 & 12.0 & 20.5 & 40.7 & 826 & 10.2 & 15.4 & 26.5 \\
\hline $\mathrm{LD}, \mathrm{U} / \mathrm{L}$ & 0.08 & Asia All & 2924 & 138 & 181 & 235 & 1291 & 142 & 184 & 240 & 1634 & 136 & 178 & 233 \\
\hline LD-JSCC, U/L & - & Japanese & 1730 & 124 & 165 & 215 & 783 & 127 & 168 & 220 & 945 & 123 & 162 & 209 \\
\hline ALP-JSCC, U/L & 0.15 & Asia All & 2873 & 34 & 56 & 90 & 1272 & 39 & 61 & 96 & 1604 & 32 & 52 & 84 \\
\hline GGT, U/L & 0.01 & Asia All & 1526 & 14 & 21 & 49 & 588 & 15 & 27 & 68 & 959 & 15 & 19 & 43 \\
\hline GGT-JSCC, U/L & - & Japanese & 1283 & 14 & 20 & 54 & 470 & 15 & 25 & 66 & 819 & 15 & 19 & 44 \\
\hline $\mathrm{CK}, \mathrm{U} / \mathrm{L}$ & 0.11 & Asia All & 2912 & 43 & 89 & 226 & 1280 & 58 & 116 & 261 & 1619 & 40 & 74 & 152 \\
\hline CK-JSCC, U/L & - & Japanese & 1712 & 42 & 86 & 192 & 781 & 60 & 108 & 263 & 938 & 40 & 71 & 135 \\
\hline AMY, U/L & 0.11 & Asia All & 2876 & 47 & 80 & 136 & 1273 & 45 & 77 & 131 & 1604 & 51 & 82 & 148 \\
\hline \multirow[t]{4}{*}{ IgG, g/L } & 0.38 & Asia All & 2885 & 9.2 & 12.9 & 18.8 & 1274 & 8.8 & 12.4 & 18.0 & 1610 & 9.5 & 13.3 & 19.1 \\
\hline & & Japanese & 1728 & 9.0 & 12.5 & 17.8 & 769 & 9.0 & 12.1 & 17.2 & 948 & 9.4 & 12.9 & 18.3 \\
\hline & & Chinese & 595 & 9.8 & 13.0 & 18.8 & 264 & 9.5 & 12.6 & 18.2 & 332 & 9.8 & 13.4 & 18.6 \\
\hline & & SE Asia & 478 & 10.8 & 14.7 & 21.7 & 190 & 10.8 & 14.1 & 21.5 & 283 & 11.4 & 15.1 & 21.8 \\
\hline \multirow[t]{4}{*}{$\lg A, g / L$} & 0.27 & Asia All & 1270 & 1.06 & 2.22 & 4.33 & 561 & 0.93 & 2.22 & 4.19 & 709 & 1.02 & 2.21 & 4.26 \\
\hline & & Japanese & 1077 & 0.94 & 2.12 & 3.96 & 491 & 0.99 & 2.23 & 4.13 & 583 & 0.95 & 2.04 & 3.80 \\
\hline & & Chinese & 390 & 1.02 & 2.34 & 4.51 & 171 & 1.01 & 2.34 & 4.39 & 219 & 1.10 & 2.38 & 4.59 \\
\hline & & SE Asia & 354 & 1.09 & 2.32 & 4.29 & 158 & 1.24 & 2.27 & 4.31 & 195 & 1.18 & 2.40 & 4.65 \\
\hline $\operatorname{Ig} M, g / L$ & 0.17 & Asia All & 2869 & 0.37 & 1.06 & 2.38 & 1267 & 0.33 & 0.84 & 1.78 & 1603 & 0.49 & 1.26 & 2.65 \\
\hline $\mathrm{C} 3, \mathrm{mg} / \mathrm{L}$ & 0.47 & Asia All & 2875 & 729 & 1007 & 1389 & 1286 & 757 & 1036 & 1427 & 1586 & 716 & 983 & 1352 \\
\hline & & Japanese & 1727 & 715 & 971 & 1301 & 771 & 738 & 1000 & 1345 & 954 & 708 & 946 & 1261 \\
\hline & & Chinese & 593 & 773 & 1064 & 1414 & 264 & 802 & 1086 & 1438 & 326 & 786 & 1040 & 1431 \\
\hline & & SE Asia & 470 & 798 & 1128 & 1526 & 198 & 819 & 1158 & 1569 & 270 & 796 & 1107 & 1475 \\
\hline $\mathrm{C} 4, \mathrm{mg} / \mathrm{L}$ & 0.41 & Asia All & 2857 & 122 & 201 & 340 & 1271 & 122 & 205 & 339 & 1601 & 112 & 192 & 339 \\
\hline & & Japanese & 1725 & 113 & 188 & 308 & 780 & 120 & 198 & 313 & 942 & 109 & 180 & 293 \\
\hline & & Chinese & 598 & 129 & 208 & 383 & 267 & 132 & 214 & 368 & 331 & 123 & 207 & 371 \\
\hline & & SE Asia & 478 & 132 & 237 & 394 & 196 & 137 & 244 & 408 & 281 & 141 & 232 & 404 \\
\hline CRP, mg/L & 0.42 & Asia All & 2849 & 0.01 & 0.38 & 2.81 & 1247 & 0.04 & 0.43 & 3.74 & 1590 & 0.01 & 0.30 & 2.57 \\
\hline & & Japanese & 1223 & 0.06 & 0.25 & 2.50 & 458 & 0.09 & 0.28 & 2.44 & 820 & 0.02 & 0.29 & 2.59 \\
\hline & & Chinese & 522 & 0.11 & 0.46 & 5.87 & 214 & 0.12 & 0.57 & 5.86 & 308 & 0.10 & 0.42 & 4.74 \\
\hline & & SE Asia & 417 & 0.11 & 0.71 & 5.71 & 156 & 0.12 & 0.85 & 4.92 & 260 & 0.11 & 0.61 & 6.66 \\
\hline TTR, mg/L & 0.24 & Asia All & 2889 & 193 & 271 & 391 & 1278 & 230 & 308 & 401 & 1612 & 186 & 246 & 331 \\
\hline Tf, g/L & 0.26 & Asia All & 2880 & 1.91 & 2.52 & 3.47 & 1271 & 1.88 & 2.42 & 3.10 & 1604 & 2.00 & 2.62 & 3.60 \\
\hline & & Japanese & 1717 & 2.01 & 2.58 & 3.52 & 775 & 1.99 & 2.48 & 3.18 & 941 & 2.04 & 2.68 & 3.66 \\
\hline & & Chinese & 600 & 1.84 & 2.45 & 3.33 & 267 & 1.76 & 2.36 & 2.95 & 330 & 1.97 & 2.56 & 3.50 \\
\hline
\end{tabular}


(Table 4 Continued)

\begin{tabular}{|c|c|c|c|c|c|c|c|c|c|c|c|c|c|c|}
\hline \multirow{2}{*}{$\frac{\text { International Unit }}{\text { Analytes, Units }}$} & \multirow[t]{2}{*}{ SDR-reg } & \multirow[t]{2}{*}{ Area } & \multirow[b]{2}{*}{$\mathbf{n}$} & \multicolumn{3}{|c|}{ Male + Female } & \multirow[b]{2}{*}{$\mathbf{n}$} & \multicolumn{3}{|r|}{ Male } & \multirow[b]{2}{*}{$\mathbf{n}$} & \multicolumn{3}{|c|}{ Female } \\
\hline & & & & LL & Me & UL & & LL & Me & UL & & LL & Me & UL \\
\hline & & SE Asia & 478 & 1.90 & 2.41 & 3.23 & 195 & 1.86 & 2.28 & 2.94 & 283 & 1.99 & 2.50 & 3.40 \\
\hline Testo, nmol/L & 0.00 & Asia All & & & & & 1269 & 10.1 & 17.4 & 28.4 & 1609 & 0.9 & 2.0 & 3.5 \\
\hline Estradiol, pmol/L & 0.00 & Asia All & & & & & 1266 & 66 & 96 & 140 & 1609 & 50 & 198 & 840 \\
\hline Progest, $\mathrm{nmol} / \mathrm{L}$ & 0.00 & Asia All & & & & & 1265 & 0.37 & 1.69 & 4.48 & 1610 & 0.1 & 3.7 & 66.5 \\
\hline Cortisol, $\mathrm{nmol} / \mathrm{L}$ & 0.21 & Asia All & 2876 & 45 & 100 & 193 & 1274 & 51 & 109 & 197 & 1605 & 41 & 92 & 190 \\
\hline
\end{tabular}

known to be related to the metabolic syndrome (TG, AST, ALT, GGT, and CRP) (Supplementary Table 4). For these five analytes, individuals consuming ethanol $>40 \mathrm{~g} /$ day and those with BMI $>26 \mathrm{~kg} / \mathrm{m}^{2}$ were excluded before deriving RIs. In addition, in derivation of RIs for IgA, individuals who reported presence of allergic conditions (allergic rhinitis, atopic dermatitis, etc.) in the questionnaire were excluded before the computation. Therefore, the number of reference individuals for those analytes was smaller.

As supplementary information, RIs were computed in total of seven ways (additionally for Taiwan, Hong Kong/ Macau, and Ho Chi Minh City), irrespective of SDR ${ }_{\text {reg }}$ values, in both international and conventional units as listed in Supplementary Table 5. Age-related reference intervals for every 10 years of age for each sex were derived both from data of the entire regions and from data limited to Japan, and were provided as Supplementary Table 6A and B.

\section{Discussion}

To avoid the potential influence of differences in occupation on test results, we recruited candidate reference individuals mostly from healthy hospital workers (84\%), with the remainder from those who worked indoors. This recruitment policy may give an impression of bias in the population. However, there are a variety of jobs in medical facilities, and the level of physical activity differs widely from one individual to another. Therefore, we believe that the target population actually represents a majority of the general population working mostly indoors. However, if we had sampled more broadly, we could not have distinguished true regional differences from those attributable to occupational environment and degree of labor. To assess the practicality of the RIs derived, further investigation may be required in each geographical area to compare the RIs with those derived based on data obtained in a setting of community health screening.
In defining reference individuals, it is also important to clarify a policy regarding how to exclude those with abnormal results due to latent diseases. Although there are many pathological conditions that may affect the test results of each analyte, we assumed that as long as the prevalence was low, inclusion of results from such cases would not have any influence on the determination of RIs targeting the central 95\% interval. In contrast, careful consideration must be given to the exclusion of those individuals with latent but highly prevalent disorders like metabolic syndrome and diabetes. For excluding abnormal results, it is not appropriate to apply clinical decision limits (CDL), generally set by consensus among clinical experts as a guide for prevention of those diseases. Such a univariate approach of excluding abnormal values results in truncation of the reference distribution.

To exclude individuals with abnormal results due to latent diseases, we applied a multivariate approach called the LAVE method $[7,9,13,14]$, which is an iterative method for optimized selection of reference individuals. In the initial iteration, it determines RIs analyte by analyte in a univariate manner without consideration of other test results. From the second iteration on, a RI for any given analyte is computed from datasets after excluding the values of individuals who have abnormal results in other analytes. By repeating this process, the RIs are progressively optimized so long as there are associations among the abnormal results. This method applies a principle similar to the one presented by Grossi et al. [16] based on the correlation among variables. In the present study, we used the RIs of 13 'basic' analytes as criteria in judging the need for exclusion: Alb, Glb, UA, Glu, AST, ALT, LD, GGT, CK, TG, HDL-C, LDL-C, and CRP. The LAVE method effectively narrows the RI when there are associations with test results of the 'basic' analytes set for exclusion criteria (see Supplementary Table 4), whereas RIs of analytes that lack such association are not affected. The LAVE method also does not cause any change in RIs of analytes for which abnormal results are rare among healthy individuals, 
such as Alb, CRE, urea, and Na. As expected, for the analytes in the lipid profile, the obtained results were different from the CDL, which have a different origin and are usually aimed at a specific group of diseases [17].

The most conspicuous finding of this study was the detection of no regionality in test results for any of the 31 analytes examined within the entire area of Japan. This implies that it is possible to share RIs nationwide in Japan for the standardized analytes that were made traceable to a reference measurement system. In contrast, when all Asian areas were included in the nested ANOVA, we observed apparent regional differences in five analytes: HDL-C, IgG, C3, C4, and CRP. In regard to inflammatory markers, we reproduced and confirmed the findings of the past two Asian studies [7, 9]: the closer the regional area is to the equator, the higher the serum concentrations of positive inflammatory markers (IgG, C3, CRP). This same tendency was also found for C4. It is assumed that exposure to infectious agents is higher in regions closer to the equator.

We were not able to identify the reason for the prominent difference in the level of HDL-C between Japan and the rest of the regions by use of 3N-ANOVA in the second Asian study. However, when we re-analyzed this separately for both sexes, we detected apparent regionality $\left(\mathrm{SDR}_{\mathrm{reg}} \geq 0.3\right)$ with the same tendency for both sexes: higher HDL-C levels in two cities in Japan compared with four other cities. However, the regionality detected in electrolytes ( $\mathrm{Na}, \mathrm{K}, \mathrm{Cl}$, and $\mathrm{Ca}$ ) and $\mathrm{LD}$ in the second study was not clearly observed this time, although there was a slight tendency for local differences in K and LD from the graph. The much smaller data size in the second study may have been responsible for the inconsistent results. This result poses a question as to the appropriateness of the cut-off value for SDR set at 0.3. In fact, when the SDR ${ }_{\text {reg }}$ was computed after separating the dataset by sex, regionality with a SDR $_{\text {reg }}$ of $\geq 0.3$ was observed in either or both of the sexes in 11 analytes, an increase of six analytes after the separation. Those analytes showing regionality only in males were urea, IgM, TTR, and Tf, and those with regionality only in females were TG and IgA. Furthermore, when we looked at the graphs (Supplementary Figure 2) showing the regional distributions, some of the test results showed a bias limited to just one or two areas. For example, test results for LD are lower in Kuala Lumpur and Jakarta, and those for ALP are higher in these two cities than elsewhere. Therefore, even when $\mathrm{SDR}_{\text {reg }}$ is well below 0.3, presence of localized bias in test results cannot be denied completely. We must also note that the number of volunteers recruited within Japan was much larger than that outside Japan, and there was no regionality within Japan. Therefore, this imbalance inevitably caused dilution of the effect of regional differences.

To adjust for regional differences in BMI and smoking and drinking habits, we applied multiple regression analyses and confirmed that none of the regionality observed in test results was influenced by the covariates. These results will be reported in the companion article to be published in this series.

Although we emphasized the presence of regionality in the above discussion, we could derive 'common' RIs valid at least in Southeast Asian countries for the majority of the analytes. As we made the test results traceable to the reference measurement procedures through careful recalibration using certified reference materials, the RIs are in a sense 'universal' as long as the constitution of the target population and the statistical methodologies do not change.

From the standpoint of traceability, we could make a valid comparison of our results with RIs for AST, ALT, and GGT made traceable to a reference measurement system that was recently derived by the IFCC C-RIDL targeting European and Asian population [6].

In conclusion, RIs which can be applicable to a wide geographical area in Asia were established for the majority of analytes with traceability to reference measuring systems, whereas regional partitioning was required for RIs of HDL-C, CRP, IgG, C3, and C4 in both sexes and for urea, TG, IgA, IgM, Tf, and TT in either of the sexes. However, no regional differences were observed in any analyte when data were limited to those from Japan.

Acknowledgments: This research has been planned collaboratively by the: 1) C-PP and C-RIDL of the IFCC; 2) the Scientific Committee of the APFCB; 3) the Working Group on the Guideline for Common Reference Interval of the JSLM (Japan Society of Laboratory Medicine); and 4) the C-PP of the JSCC. This study was supported by the C-RIDL of the IFCC.

Research funds used included a Scientific Research Fund (No. 21406015: 2009-2011) provided by Japan Society for the Promotion of Science; a Research Promotion Project Fund of the JSLM (2008-2009); and a Scientific Research Fund of the APFCB.

Most of the reagents and labor required for testing such a large number of analytes were generously offered by BC. Additional reagents were provided by Nittobo Co. The sampling equipment (vacuum sample tubes, needles, and holders) were supplied by BD. Other expenses, such as serum container tubes, boxes, and ID labels, were purchased with the Scientific Research Funds offered by the APFCB. 
The value-assigned specimens for the four steroid hormones were provided by L. Siekmann. A set of three fresh-frozen $\left(-80^{\circ} \mathrm{C}\right)$ pooled sera for each of five enzymes (AST, ALT, LDH, GGT, and CK) were assigned values by the laboratories of F. Ceriotti and M. Panteghini based on the reference measurement procedures.

We are grateful to the participating laboratories and their staffs for their contributions to this study. The collaborating laboratories outside Japan were: 1) Gangnam Severance Hospital, Yonsei University, Seoul; 2) Kangbuk Samsung Hospital, Sungkyunkwan University, Seoul; 3) Myongji Hospital, Kwandong University College of Medicine, Gyeonggi-do; 4) Peking University First Hospital, Beijing; 5) Mackay Memorial Hospital, Taipei; 6) Cathay General Hospital, Taipei; 7) Yuan Ching Clin Laboratory, Taipei; 8) National Cheng Kung University, Tainan; 9) Chi-Mei Medical Center, Tainan; 10) Prince of Wales Hospital, Chinese University of Hong Kong, Hong Kong; 11) Macau Institute for Applied Research in Medicine and Health, Macau University of Science and Technology, Macau; 12) Medic-Lab, Ho Chi Minh City, 13) National Heart Institute, Kuala Lumpur; 14) Gleneagles Intan Medical Centre, Kuala Lumpur; and 15) Prodia Clinical Laboratory, Jakarta.

The collaborating laboratories within Japan included: 16) Keiyu Corp. Yoshida Hospital, Asahikawa; 17) Hokkaido University, Sapporo; 18) Kishimoto Clinical Laboratory, Tomakomai; 19) Iwate Medical School, Morioka; 20) Hirosaki University, Hirosaki; 21) Hachinohe RedCross Hospital, Hachinohe; 22) Hachinohe City Hospital, Hachinohe; 23) Chiba Cardiovascular Center, Ichihara; 24) Chiba University, Chiba; 25) Funabashi Municipal Medical Center, Funabashi; 26) Tokyo Medical and Dental University Chiba Hospital, Chiba; 27) Tokyo University, Tokyo; 28) Toho University, Tokyo; 29) Shinshu University, Matsumoto; 30) Matsumoto JAM Medical Center, Matsumoto; 31) Ohmachi Municipal Hospital, Omachi; 32) Yamanashi University, Kofu; 33) Fukui University, Fukui; 34) Kanazawa Medical School, Kanazawa; 35) SRL-Kanazawa, Kanazawa; 36) Mie University, Tsu; 37) Nagoya University, Nagoya; 38) Anjo Kosei Hospital, Anjo; 39) Fujita Health University, Toyoake; 40) Osaka University, Suita; 41) Tenri Hospital, Tenri; 42) Osaka Municipal University, Osaka;
43) National Cardiovascular Center, Suita; 44) Kawasaki Medical School, Kurashiki; 45) Kurashiki Central Hospital, Kurashiki; 46) Okayama University, Okayama; 47) Okayama Medical Laboratory, Kurashiki; 48) Matsuda Hospital, Kurashiki; 49) Hiroshima University, Hiroshima; 50) Yamaguchi University, Ube; 51) Tokuyama Central Hospital, Shunan; 52) Yamaguchi Prefectural Medical Center, Hofu; 53) Saiseikai Yamaguchi Hospital, Yamaguchi; 54) Saint Hill Hospital, Ube; 55) Kochi Medical School Hospital, Nangoku; 56) Kochi Red-Cross Hospital, Kochi; 57) Kochi National Hospital, Kochi; 58) Kumamoto University, Kumamoto; 59) CIS Hitoyoshi Laboratories, Hitoyoshi; 60) Tsuruta Memorial Clinic and Cancer Diagnostic Center, Miyazaki; 61) Eiwa Corp. Terada Hospital, Isa; 62) Ryukyu University, Naha; and 63) Adventist Medical Center, Naha.

This study is also indebted to Professor Giampaolo Merlini (University Hospital San Matteo), Professor Yoshihisa Itoh (Asahikawa Medical University), Emeritus Professor Takeshi Kanno (Hamamatsu University School of Medicine), Emeritus Professor Tadashi Kawai (Jichi University School of Medicine), Dr. Katsuhiko Kuwa (National Metrology Institute of Japan), Professor Susumu Osawa (Kyushu University School of Medicine), and Professor Shigemi Hosogaya (Kagawa Prefectural College of Health Sciences), who gave invaluable advice and encouragement to promote and carry out this study. Lastly, we are very grateful to Dr. Joseph Henny, Dr. Jose M. Queralto, and Dr. James C. Boyd as members of C-RIDL who gave us invaluable comments during a series of discussions on the scientific implications of this study.

\section{Conflict of interest statement}

Authors' conflict of interest disclosure: The authors stated that there are no conflicts of interest regarding the publication of this article.

Research funding: None declared.

Employment or leadership: None declared.

Honorarium: None declared.

Received June 29, 2012; accepted January 8, 2013

\section{References}

1. Panteghini M, Forest JC. Standardization in laboratory medicine: new challenges. Clin Chim Acta 2005;355:1-12.

2. Directive 98/79/EC of European Parliament and of the Council of 27 October 1998 on in vitro diagnostics medical devices. Offic J Eur Commun 1998;L331:1-37.
3. International Organization for Standardization. Medical Laboratories - Particular requirements for quality and competence ISO 15189. Geneva: ISO, 2007.

4. Rustad P, Felding P, Lahti A, Hyltoft Petersen P. Descriptive analytical data and consequences for calculation of common 
reference intervals in the Nordic Reference Interval Project 2000. Scand J Clin Lab Invest 2004;64:343-70.

5. Kinoshita S, Toyofuku M, lida H, Wakiyama M, Kurihara M, Nakahara M, et al. Standardization of laboratory data and establishment of reference intervals in the Fukuoka Prefecture: a Japanese perspective. Clin Chem Lab Med 2001;39:256-62.

6. Ceriotti F, Henny J, Queraltó J, Ziyu S, Özarda Y, Chen B, et al. Common reference intervals for aspartate aminotransferase (AST), alanine aminotransferase (ALT) and $\gamma$-glutamyl transferase (GGT) in serum: results from an IFCC multicenter study. Clin Chem Lab Med 2010;48:1593-601.

7. Ichihara K, Itoh Y, Min WK, Yap SF, Lam CW, Kong XT, et al. Diagnostic and epidemiological implications of regional differences in serum concentrations of proteins observed in six Asian cities. Clin Chem Lab Med 2004;42:800-9.

8. Johnson AM, Whicher JT, Ledue TB, Carlström A, Itoh Y, Petersen $\mathrm{PH}$. Effect of a new international reference preparation for proteins in human serum (certified reference material 470) on results of the College of American Pathologists Surveys for plasma proteins. Arch Pathol Lab Med 2000;124:1496-501.

9. Ichihara K, Itoh Y, Lam CW, Poon PM, Kim JH, Kyono H, et al. Sources of variation of commonly measured serum analytes among 6 Asian cities and consideration of common reference intervals. Clin Chem 2008;54:356-65.

10. Ceriotti F. Prerequisites for use of common reference intervals. Clin Biochem Rev 2007;28:115-21.

11. Sokal RR, Rohlf FJ. Linear regression (Model II regression). In Biometry: the principles and practice of statistics in biological research, 3rd ed. San Francisco: W. H. Freeman, 1995:541-9.

12. Andrew J. Bohonak. RMA: software for reduced major axis regression. Available from: http://www.bio.sdsu.edu/pub/ andy/RMA.html. Accessed 23 April, 2012.

13. Ichihara K, Boyd JC; IFCC Committee on Reference Intervals and Decision Limits (C-RIDL). An appraisal of statistical procedures used in derivation of reference intervals. Clin Chem Lab Med 2010;48:1537-51.

14. Ichihara K, Kawai T. Determination of reference intervals for 13 plasma proteins based on IFCC international reference preparation (CRM470) and NCCLS proposed guideline (C28-P,1992): trial to select reference individuals by results of screening tests and application of maximal likelihood method. J Clin Lab Anal 1996;10:110-7.

15. Harris EK, Boyd JC. Statistical basis of reference values in laboratory medicine. New York: Marcel Dekker, 1995.

16. Grossi E, Colombo R, Cavuto S, Franzini C. The REALAB Project: a new method for the formulation of reference intervals based on current data. Clin Chem 2005;51:1232-40.

17. Ceriotti F, Henny J. Are my laboratory results normal? Considerations to be made concerning reference intervals and decision limits. eJIFCC 2008; Vol 19 no 2. Available from: http://www.ifcc.org/ifcc-communications-publications-division(cpd)/ifcc-publications/ejifcc-(journal)/e-journal-volumes/ vol-19-n\%C2\%B0-2/are-my-laboratory-results-normal-consid erations-to-be-made-concerning-reference-intervals-and-de/. Accessed 23 April, 2012. 